\title{
CD40 agonists-a promising new treatment for pancreatic cancer?
}

Treatment with the fully human CD40 agonist antibody CP-870,893 can achieve substantial regression of tumors in some patients with inoperable pancreatic ductal adenocarcinoma (PDAC), according to a recent study. "[This] tumor ... generates marked immune suppression so our goal was to reverse the process using the CD40 [agonist]," explains Robert Vonderheide, the study's corresponding author. As CD40 activation promotes T-cell-dependent antitumor immunity, the patients also received gemcitabine, which enhances tumor antigen presentation.

Median progression-free survival for the 21 treated patients was 5.6 months, nearly double that seen with gemcitabine alone (2.3 months). Median overall survival was 7.4 months (versus 5.7 months with gemcitabine alone). Tumor regression was observed in four of the 21 patients. However, the researchers were surprised to find that biopsy samples of tumors that regressed contained macrophages, but no leukocytes.

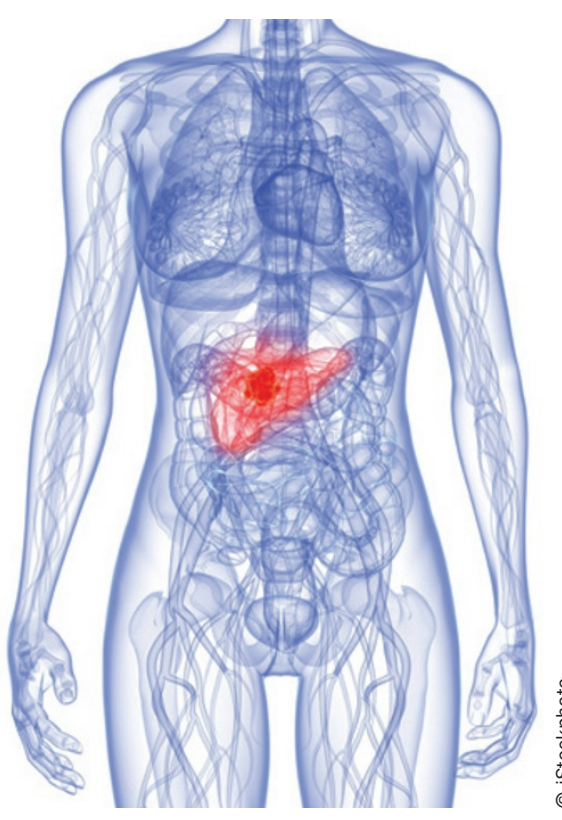

resulted in tumor regression in 30\% of mice. Interestingly, FGK45 induced tumor regression even in mice lacking $\mathrm{CD}^{+}$or $\mathrm{CD}^{+}$or both types of $\mathrm{T}$ cell. However, depletion of macrophages by treatment with clodronate-encapsulated liposomes abrogated the beneficial effects of FGK45. The investigators then showed that treatment with FGK45 led to infiltration of tumor tissue by CD40activated macrophages, which resulted in degradation of the tumor stroma.

"We are now focused on improving this therapy [on the basis] of insights we have from the laboratory studies and to bring the best ideas forward to patients with both pancreatic cancer and other tumors," concludes Vonderheide.

\section{Shreeya Nanda}

The researchers conducted futher studies in mouse models of PDAC, which showed that administration of the CD40 agonist antibody FGK45 and gemcitabine
Original article Beatty, G. L. et al. CD40 agonists alter tumor stroma and show efficacy against pancreatic carcinoma in mice and humans. Science 331, 1612-1616 (2011) 